\title{
$\Lambda$ is Coming: Parametrizing Freezing Fields
}

\author{
Eric V. Linder ${ }^{1,2}$ \\ ${ }^{1}$ Berkeley Center for Cosmological Physics $\&$ Berkeley Lab, \\ University of California, Berkeley, CA 94720, USA \\ ${ }^{2}$ Energetic Cosmos Laboratory, Nazarbayev University, Astana, Kazakhstan 010000
}

(Dated: February 10, 2017)

\begin{abstract}
We explore freezing dark energy, where the evolution of the field approaches that of a cosmological constant at late times. We propose two general, two parameter forms to describe the class of freezing field models, in analogy to ones for thawing fields, here based on the physics of the flow parameter or the calibrated $w-w^{\prime}$ phase space. Observables such as distances and Hubble parameters are fit to within $0.1 \%$, and the dark energy equation of state generally to within better than $1 \%$, of the exact numerical solutions.
\end{abstract}

\section{INTRODUCTION}

Cosmic acceleration of the expansion of the universe can be treated as arising from an effective scalar field; this holds even if the physical origin is actually, say, from a higher dimensional braneworld. At the level of the Friedmann equations, the expansion $a(t)$ can be equivalently described by a dark energy equation of state $w(a)$, modulo the matter density.

The physics of an observationally viable universe - one with a long period of matter domination in which density perturbations can grow and then a recent period of cosmic acceleration - group the acceptable scalar field behaviors into two classes: thawing fields and freezing fields that lie in distinct regions of the $w-w^{\prime}$ phase space [1-4]. Here a prime denotes $d / d \ln a$. These classes are related to the competition between the driving term of the steepness of the potential and the friction of the Hubble expansion. The separation between the classes is enforced by avoidance of fine tuning in the field, that the acceleration of the field will generically not be so exquisitely balanced that it vanishes.

Across these classes a highly successful general description is provided in terms of two parameters, $w_{0}$ and $w_{a}$, corresponding to the present dark energy of state parameter and a measure of its time variation. This was first derived in terms of exact solutions of the Klein-Gordon equation of motion [5] and later quantified as providing $0.1 \%$ accuracy on observable quantities [4]. For some purposes, though, one might be interested in going beyond observables and seeking an improved description of the equation of state $w(a)$ itself.

An equation of state description for the class of thawing fields has been treated in depth in the literature [611]. Most recently, a one parameter form simplifying the general algebraic thawing expression of [7] has been demonstrated to have $0.3 \%$ accuracy in $w(a)$ [12]. Freezing field models however have been more problematic, due to their greater diversity in initial conditions and evolution. Here we address the issue of parametrizing the equation of state for the class of freezing models.

Section II discusses the methodology, and we present the results in Sec. III, before concluding in Sec. IV.

\section{METHODOLOGY}

Freezing fields of interest start in the matter dominated epoch (by which is meant either nonrelativistic matter or radiation) with the field rolling down a steep potential. At late times (in the future) the field freezes as the field enters the shallow region of the potential, and the field acts like a cosmological constant, asymptotically approaching a de Sitter state (though the field motion may never vanish completely at finite times). Potentials of interest do not have a nonzero minimum, i.e. an intrinsic cosmological constant.

Early freezing models date back to the exponential and inverse power law potentials [13-16] and were particularly interesting for having an attractor behavior that brought the field from a wide variety of initial conditions onto a tracker trajectory at high redshift where the equation of state became constant. One problem with this was the difficulty of moving the field sufficiently quickly off the tracker so that it could attain a sufficiently negative equation of state $w \approx-1$ near the present. The potential needed to be modified to allow this to happen, e.g. within the supergravity inspired approach of [17].

An interesting discovery was that higher dimensional braneworld models [18] and more phenomenological generalizations by adding a term $H^{\alpha}$ to the Friedmann expansion equation [19] (with the DGP braneworld corresponding to $\alpha=1$ ) acted in a very similar way to freezing scalar fields, and indeed one could write down an effective potential [20]. At early times these behave like an inverse power law potential $V \sim \phi^{-n}$ with index $n=2 \alpha /(2-\alpha)$. At late times, such freezers approach $w=-1$ along the phase space trajectory $w^{\prime}=3 w(1+w)$, the lower boundary of the freezing region. This corresponds to a potential

$$
V(\phi)=V_{\infty}\left[1+\frac{3}{8}\left(\phi_{\infty}-\phi\right)^{2}\right],
$$

where the field asymptotically freezes to the value $\phi_{\infty}$ (while this nominally has a nonzero minimum, it is only an effective potential and there is no true cosmological constant, e.g. in Minkowski space).

Thus the variety of potentials means that there is no expectation that attempting to find a common 
parametrization of freezing field potentials should be successful. Indeed, there are clear differences between inverse power law and supergravity inspired potentials. Thus, we instead focus on capturing the key physics common to different classes of potentials.

One approach is to follow the physics in terms of the flow parameter [20]

$$
F(a) \equiv \frac{1+w(a)}{\Omega_{\phi}(a)\left(V_{\phi} / V\right)^{2}},
$$

where $\Omega_{\phi}(a)$ is the fractional dark energy density and $V_{\phi}=d V / d \phi$. The flow parameter was shown to be conserved through the matter dominated era, with the value $4 / 27$ for the thawing class and $1 / 3$ for the freezing class. During the present accelerating epoch $F$ slowly deviates from these asymptotic values as the dark energy density increases. The flow parameter is directly related to the dark energy phase space evolution and so given a parametrization for $F$ one can derive $w(a)$, from

$$
w^{\prime}=-3\left(1-w^{2}\right)\left[1-\frac{1}{\sqrt{3 F}}\right] .
$$

Another approach to consider is based on the phenomenological calibration of the phase space trajectories exhibited in [4]. This showed great success in taking the diversity of equation of state behaviors and defining "stretching parameters" (leading to particular versions of $w_{0}$ and $w_{a}$ ) that focused the models into narrow bands or families. One might parametrize the freezing models according to band and location along the band.

We now discuss these two approaches - the flow and calibration approaches - in more detail.

\section{A. Flow Parametrization}

We know that the physics of the long, matter dominated epoch forces the field evolution into certain paths. This causes the combination of dark energy density, equation of state, and steepness of the potential to be interrelated in such a way as to keep constant the combination in the flow parameter $F$. If we focus on tracker freezing fields, as the most attractive due to their insensitivity to initial conditions, then the early equation of state is constant, i.e. the dark energy density $\rho_{\phi} \sim a^{-3(1+w)}$, and so by Eq. (3) we see that $F=1 / 3$ during matter domination.

As the dark energy density grows, $F$ begins to increase from this constant, but slowly - see Fig. 4 of [20] - with the deviation proportional to the fractional dark energy density $\Omega_{\phi}$. Since dark energy has only become significant within the last e-fold of cosmic expansion, and is not fully dominant today, a reasonable ansatz for the flow parametrization is

$$
F(a)=\frac{1}{3}\left[1-\Omega_{\phi}(a)\right]+b \Omega_{\phi}(a),
$$

where $b$ is a constant parametrizing the specific freezing model. In the matter dominated epoch this expression reduces to $F=1 / 3$ as desired, and $F$ again goes to a constant in the future. Since we do not have observations in the future, we do not force $b$ to a particular value (e.g. for all braneworld models it would be $4 / 3$ ), but leave it as a fitting parameter to observable data.

Note that $F=1 / 3$ does not fully characterize the initial conditions as this simply leads to $w^{\prime}=0$ at early times, without determining the specific value of $w$ in the high redshift tracking regime. This is then an additional parameter $w_{\infty}$, where in the case of early time inverse power law potential behavior $w_{\infty}=-2 /(n+2)$. The two parameters of this ansatz are then $b$ and either $w_{\infty}$ or $n$. We will keep the second parameter as $w_{\infty}$ since this is somewhat more generic.

To solve the evolution we have a system of coupled equations of motion,

$$
\begin{aligned}
w^{\prime} & =-3\left(1-w^{2}\right)\left[1-\frac{1}{\sqrt{3 F}}\right] \\
\Omega_{\phi}^{\prime} & =-3 w \Omega_{\phi}\left(1-\Omega_{\phi}\right) .
\end{aligned}
$$

One can write a closed form solution for $w(a)$, given by

$$
\begin{aligned}
w & =\frac{C-1}{C+1} \\
C & =\frac{1+w_{i}}{1-w_{i}}\left(\frac{a}{a_{i}}\right)^{-6} e^{2 \sqrt{3} \int_{a_{i}}^{a} d \ln a^{\prime} F\left(a^{\prime}\right)^{-1 / 2}} .
\end{aligned}
$$

If one adopts a perturbative expansion about the high redshift value of the form $F(a)=F_{0}+F_{1} a^{s}$, e.g. since $\Omega_{\phi} \sim a^{-3\left(1+w_{\infty}\right)}$ at high redshift, one can solve this analytically [21] but since we are interested in times when the dark energy density becomes dominant we will work with the form Eq. (4) and the numerical solution.

The flow parametrization has the useful property that it is closely connected (much more so than the potential) to what we want: the dark energy equation of state $w(a)$.

\section{B. Calibration Parametrization}

An even more direct approach is to parametrize $w(a)$ explicitly. This is of course exactly what the standard $w_{0}-w_{a}$ form does, but here we are focusing specifically on the freezing class and seek increased accuracy. One application of $w_{0}-w_{a}$ did this with an eye on the distanceredshift relation, in [4], where the phase space trajectories of freezing models were calibrated through a stretching relation

$$
w_{a}^{\text {calib }} \equiv-w^{\prime}\left(a_{\star}\right) / a_{\star} .
$$

Adopting the value of the temporal stretching parameter $a_{\star}=0.85$ was found to calibrate the freezing field models into tight family bands, and the resulting

$$
w(a)=w_{0}+w_{a}^{\text {calib,d }}(1-a)
$$


gave accurate reconstruction of the observable distances and Hubble parameters at the $0.1 \%$ level.

Here we will parametrize the phase space to fit $w(a)$ itself. We will find in the next section that this works very similarly to the distance calibration, but here $w_{a}$ is defined by choosing the stretching to calibrate the entire evolution $w(a)$. This results in the stretching parameter becoming $a_{\star}=0.82$. Thus this approach is also a parametrization with two free parameters, $w_{0}$ and $w_{a}^{\text {calib,w }}$, where the superscript calib,w indicates $w_{a}$ is fit to $w(a)$ rather than to distance or to $w_{a}=-2 w^{\prime}(z=1)$ or some similar constraint.

\section{RESULTS}

To investigate how well the flow parametrization and calibration parametrization can reconstruct the true equation of state evolution $w(a)$ we consider several typical freezing models.

The inverse power law (IPL) potential $[14,16] V(\phi)=$ $V_{\star} \phi^{-n}$ tracks at high redshift with a dark energy equation of state $w_{\infty}=-2 /(n+2)$. However, it does not evolve rapidly toward $w \approx-1$ as the dark energy density grows, and so does not accord well with current observations unless $n \ll 1$.

To ameliorate this, the supergravity inspired (SUGRA) potential [17] $V(\phi)=V_{\star} \phi^{-n} e^{\phi^{2} /\left(2 M_{p}^{2}\right)}$ enables values much closer to $w \approx-1$ to be attained by the present, despite acting like IPL at high redshift. For the IPL and SUGRA potentials, we solve the Klein-Gordon equation of motion numerically (by a fourth order Runge-Kutta technique) to obtain $w(a)$.

A more phenomenological model of interest is when the dark energy contribution to the Friedmann expansion equation takes the form of a $H^{\alpha}$ modification [19], where $H$ is the Hubble parameter. The case where $\alpha=1$ corresponds to higher dimensional braneworld DGP gravity [18]; this has the present equation of state too far from $w=-1$ to be viable, but $w_{0}$ approaches -1 for smaller $\alpha$ (with $\alpha=0$ corresponding to the cosmological constant).

The $H^{\alpha}$ model evolution can be solved analytically, with

$$
\begin{aligned}
a & =\left(\frac{\Omega_{\phi}}{\Omega_{\phi, 0}}\right)^{2 /[3(2-\alpha)]}\left(\frac{1-\Omega_{\phi, 0}}{1-\Omega_{\phi}}\right)^{1 / 3} \\
w & =-\left(\frac{2-\alpha}{2-\alpha \Omega_{\phi}}\right)
\end{aligned}
$$

where a subscript 0 denotes the present.

For each model we will choose values of $w_{\infty}$ (or equivalently $n$ or $\alpha$ ) and then find the values of the free parameter $b$ from Eq. (4) that best approximate the exact $w(a)$ functions. Figure 1 illustrates that the flow parametrization provides excellent approximations, better than $1 \%$ in $w(a)$ for the viable models not too far from $w \approx-1$. Even for $w_{\infty}=-0.75$ cases, $w(a)$ is reconstructed to better than $1 \%$ over almost all of cosmic history.

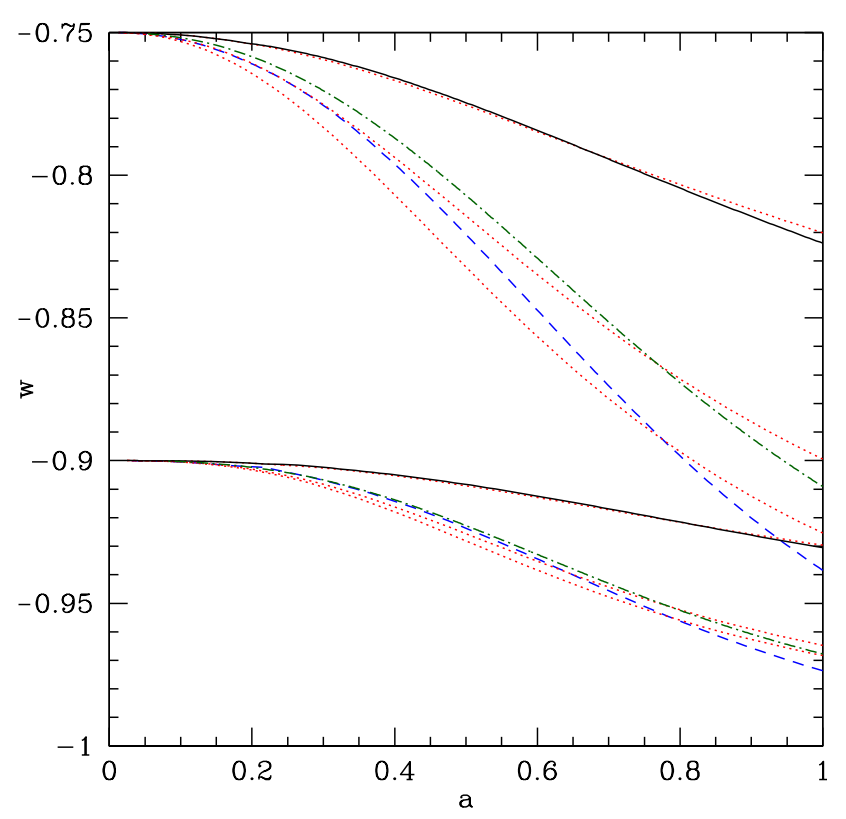

FIG. 1. Flow parametrization (dotted, red) for $w(a)$ compared to exact results for IPL (solid, black), SUGRA (dashed, blue), and $H^{\alpha}$ (dot-dashed, green) cases.

We repeat the comparison of the exact numerical results with the approximation given by the calibration parametrization in Fig. 2. In order to reflect the physics of the tracker freezing fields at high redshifts, we fix $w(a<0.25)=w(a=0.25)$. Again the parametrization is highly successful, accurate at the subpercent level, and at the $0.01 \%-0.1 \%$ level on the observables of the distances and Hubble parameters as a function of redshift.

Tables I and II list the maximum deviations in $w(a)$, and in the observables of distance $d(z)$ and Hubble parameter $H(z)$, over all redshifts, for the flow parametrization and the calibration parametrization respectively. Deviations in the distance to cosmic microwave background last scattering are in all cases near the $10^{-4}$ level. Note that freezing models consistent with observations would generally have $w_{\infty} \lesssim-0.9$; indeed the models with $w_{\infty}=-0.75$ have distances to $\mathrm{CMB}$ last scattering more than $1 \%$ different from a $\Lambda$ model with the same present matter density (and one would have to shift the matter density by $\sim 0.04$ to get agreement in most cases).

\section{CONCLUSIONS}

Dark energy evolution and the ensuing cosmic acceleration is a competition between the steepness of the (effective) potential and the Hubble friction of the cosmic expansion. This, together with the long evolution through the matter dominated epoch, naturally defines two classes of dark energy: thawing models that depart from a frozen, cosmological constant like state and freez- 


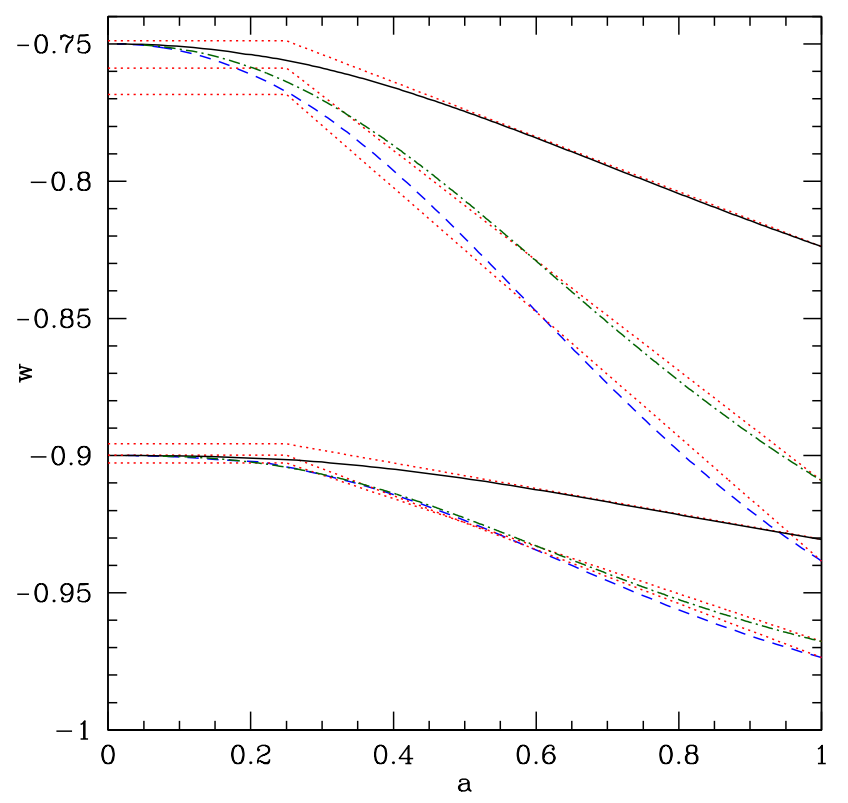

FIG. 2. Calibration parametrization (dotted, red) for $w(a)$ compared to exact results for IPL (solid, black), SUGRA (dashed, blue), and $H^{\alpha}$ (dot-dashed, green) cases.

\begin{tabular}{lccc}
\hline Model & $\delta d / d$ & $\delta H / H$ & $\delta w / w$ \\
\hline IPL $\left(w_{\infty}=-0.9\right)$ & $0.03 \%$ & $0.06 \%$ & $0.1 \%$ \\
SUGRA $\left(w_{\infty}=-0.9\right)$ & $0.04 \%$ & $0.04 \%$ & $0.5 \%$ \\
BW $\left(w_{\infty}=-0.9\right)$ & $0.02 \%$ & $0.04 \%$ & $0.3 \%$ \\
IPL $\left(w_{\infty}=-0.75\right)$ & $0.04 \%$ & $0.04 \%$ & $0.4 \%$ \\
SUGRA $\left(w_{\infty}=-0.75\right)$ & $0.1 \%$ & $0.07 \%$ & $1.4 \%$ \\
BW $\left(w_{\infty}=-0.75\right)$ & $0.08 \%$ & $0.07 \%$ & $1.1 \%$
\end{tabular}

TABLE I. Accuracy of flow parametrization in fitting the exact distances, Hubble parameters, and dark energy equation of state for various dark energy models. These numbers represent the maximum deviation over all redshifts.

ing models that approach cosmological constant behavior. This description holds over a wide range of models for canonical scalar fields and modified gravity that can be viewed as an effective dark energy.

A general treatment for both classes is given by the $w_{0}-w_{a}$ phase space parametrization, demonstrated to be accurate in the observables to $0.1 \%$. One can also focus on the dark energy equation of state function $w(a)$ itself. While the thawing class has been relatively tractable in such treatment, the freezing class has not. Here we investigated parametrization of the freezing class at the same accuracy as achieved on $w(a)$ for the thawing class. This is more complicated in that the dark energy at early times does not have $w=-1$, while at present dark energy is not completely dominant. $\Lambda$ is coming, but it is not yet here.

We demonstrate two approaches to parametrization of $w(a)$ for freezing fields, both with reasonably physical

\begin{tabular}{lccc}
\hline Model & $\delta d / d$ & $\delta H / H$ & $\delta w / w$ \\
\hline IPL $\left(w_{\infty}=-0.9\right)$ & $0.01 \%$ & $0.02 \%$ & $0.6 \%$ \\
SUGRA $\left(w_{\infty}=-0.9\right)$ & $0.03 \%$ & $0.04 \%$ & $0.4 \%$ \\
BW $\left(w_{\infty}=-0.9\right)$ & $0.03 \%$ & $0.04 \%$ & $0.3 \%$ \\
IPL $\left(w_{\infty}=-0.75\right)$ & $0.02 \%$ & $0.03 \%$ & $0.9 \%\left(0.5 \%_{z<2}\right)$ \\
SUGRA $\left(w_{\infty}=-0.75\right)$ & $0.07 \%$ & $0.1 \%$ & $2.4 \%\left(0.8 \%_{z<3}\right)$ \\
BW $\left(w_{\infty}=-0.75\right)$ & $0.05 \%$ & $0.07 \%$ & $1.2 \%\left(0.6 \%_{z<3}\right)$
\end{tabular}

TABLE II. Accuracy of calibration parametrization $w_{0^{-}}$ $w_{a}^{\text {calib,w }}$ in fitting the exact distances, Hubble parameters, and dark energy equation of state for various dark energy models. These numbers represent the maximum deviation over all redshifts, with in some cases parenthetical quantities representing deviations in all but the early universe.

levels of motivation. The flow parametrization builds on the physics of dark energy evolution during the long epoch of matter domination when the flow parameter is constant, and parametrizes its deviation as dark energy grows in influence. For observationally viable models (not too far from $w=-1$ ), it achieves better than $0.5 \%$ accuracy on $w(a)$ over all cosmic history, as well as reconstructing observable distances and Hubble parameters to better than $0.06 \%$ accuracy. Even for excursions out to $w=-0.75$ the accuracy on $w(a)$ remains near the $1 \%$ level and on the observables to $0.1 \%$.

The second approach is the calibration parametrization, using the known concept of calibration of the $w-w^{\prime}$ phase space by stretching the time variable. This gives the familiar $w_{0}-w_{a}$ parametrization but here $w_{a}$ is defined by choosing the stretching such that it calibrates the entire evolution $w(a)$. Note that the stretching is chosen to be model independent, i.e. it is fixed for the entire freezing class. This is successful (with the imposed model independent leveling at high redshift) at better than $0.6 \%$ accuracy in $w(a)$ and $0.04 \%$ accuracy in the observables for observationally viable models, and remains at better than $1 \%$ accuracy on $w(a)$ for excursions out to $w=-0.75$ for $z<3$.

Both approaches use simple, two parameter fits just like $w_{0}-w_{a}$. (Recall that the thawing class actually attained excellent accuracy even with a one parameter fit.) For the flow parametrization this is $\left\{w_{\infty}, b\right\}$, which provides information on the high redshift, tracking state; for the calibration parametrization this is $\left\{w_{0}, w_{a}^{\text {calib,w }}\right\}$, very similar to the standard, general parametrization. One can easily derive $w_{\infty}=w_{0}+0.75 w_{a}^{\text {calib, } w}$ in this approach as well.

If our universe is such that indeed $\Lambda$ is coming, either of these approaches gives a way to characterize accurately, in a fairly model independent manner, the dark energy equation of state evolution as well as the observables.

\section{ACKNOWLEDGMENTS}

I thank Zack Slepian for useful conversations. This work is supported in part by the Energetic Cosmos Lab- 
[1] I. Zlatev, L. Wang, P.J. Steinhardt, Phys. Rev. Lett. 82, 896 (1999) [arXiv:astro-ph/9807002]

[2] R.R. Caldwell, E.V. Linder, Phys. Rev. Lett. 95, 141301 (2005) [arXiv:astro-ph/0505494]

[3] E.V. Linder, Phys. Rev. D 73, 063010 (2006) [arXiv:astro-ph/0601052]

[4] R. de Putter, E.V. Linder, JCAP 0810, 042 (2008) [arXiv:0808.0189]

[5] E.V. Linder, Phys. Rev. Lett. 90, 091301 (2003) [arXiv:astro-ph/0208512]

[6] R. Crittenden, E. Majerotto, F. Piazza, Phys. Rev. Lett. 98, 251301 (2007) [arXiv:astro-ph/0702003]

[7] E.V. Linder, Gen. Rel. Grav. 40, 329 (2008) [arXiv:0704.2064]

[8] R.J. Scherrer, A.A. Sen, Phys. Rev. D 77, 083515 (2008) [arXiv:0712.3450]

[9] S. Dutta, R.J. Scherrer, Phys. Rev. D 78, 123525 (2008) [arXiv:0809.4441]

[10] T. Chiba, Phys. Rev. D 79, 083517 (2009) (err. Phys. Rev. D 80, 109902) [arXiv:0902.4037]
[11] Z. Huang, J.R. Bond, L. Kofman, Ap. J. 726, 64 (2011) [arXiv:1007.5297]

[12] E.V. Linder, Phys. Rev. D 91, 063006 (2015) [arXiv:1501.01634]

[13] C. Wetterich, Nucl. Phys. B 302, 668 (1988)

[14] B. Ratra and P.J.E. Peebles, Phys. Rev. D 37, 3406 (1988)

[15] P.G. Ferreira, M. Joyce, Phys. Rev. D 58, 023503 (1998) [arXiv:astro-ph/9711102]

[16] A.R. Liddle, R.J. Scherrer, Phys. Rev. D 59, 023509 (1999) [arXiv:astro-ph/9809272]

[17] P. Brax and J. Martin, Phys. Lett. B 468, 40 (1999) [arXiv:astro-ph/9905040]

[18] G. Dvali, G. Gabadadze, M. Porrati, Phys. Lett. B 485, 208 (2000) [arXiv:hep-th/0005016]

[19] G. Dvali, M.S. Turner 2003, arXiv:astro-ph/0301510

[20] R.N. Cahn, R. de Putter, E.V. Linder, JCAP 0811, 015 (2008) [arXiv:0807.1346]

[21] M. Cortês, E.V. Linder, Phys. Rev. D 81, 063004 (2010) [arXiv:0909.2251] 